\title{
3D Bioprinting Human Induced Pluripotent Stem Cell-Derived Neural Tissues Using a Novel Lab-on-a-Printer Technology
}

\author{
Laura de la Vega ${ }^{1}$, Diego A. Rosas Gómez ${ }^{2}$, Emily Abelseth ${ }^{3}$, Laila Abelseth ${ }^{3}$, \\ Victor Allisson da Silva ${ }^{4}$ and Stephanie M. Willerth $1,3,5, *$ (D) \\ 1 Department of Mechanical Engineering, University of Victoria, BC V8P 5C2, Canada; laura.dlvr@gmail.com \\ 2 School of Engineering and Sciences, Monterrey Institute of Technology and Higher Education, \\ Mexico City 14380, Mexico; A01650992@itesm.mx \\ 3 Biomedical Engineering Program, University of Victoria, BC V8P 5C2, Canada; \\ emilyabelseth@gmail.com (E.A.); lailaabelseth@gmail.com (L.A.) \\ 4 Center of Mathematics, Computing and Cognition, Federal University of ABC, São Paulo 09606-045, Brazil; \\ victor.allisson@aluno.ufabc.edu.br \\ 5 Division of Medical Sciences, University of Victoria, BC V8P 5C2, Canada \\ * Correspondence: willerth@uvic.ca; Tel.: +1-250-721-7303
}

Received: 29 October 2018; Accepted: 23 November 2018; Published: 28 November 2018

check for updates

Featured Application: Here, we detail a novel high-throughput method for printing neural tissues derived from human induced pluripotent stem cells (hiPSCs), which can potentially serve as a novel tool for drug screening.

Abstract: Most neurological diseases and disorders lack true cures, including spinal cord injury (SCI). Accordingly, current treatments only alleviate the symptoms of these neurological diseases and disorders. Engineered neural tissues derived from human induced pluripotent stem cells (hiPSCs) can serve as powerful tools to identify drug targets for treating such diseases and disorders. In this work, we demonstrate how hiPSC-derived neural progenitor cells (NPCs) can be bioprinted into defined structures using Aspect Biosystems' novel RX1 bioprinter in combination with our unique fibrin-based bioink in rapid fashion as it takes under $5 \mathrm{~min}$ to print four tissues. This printing process preserves high levels of cell viability $(>81 \%)$ and their differentiation capacity in comparison to less sophisticated bioprinting methods. These bioprinted neural tissues expressed the neuronal marker, $\beta$ T-III (45 $\pm 20.9 \%$ ), after 15 days of culture and markers associated with spinal cord (SC) motor neurons (MNs), such as Olig2 (68.8 $\pm 6.9 \%)$, and HB9 $(99.6 \pm 0.4 \%)$ as indicated by flow cytometry. The bioprinted neural tissues expressed the mature MN marker, ChaT, after 30 days of culture as indicated by immunocytochemistry. In conclusion, we have presented a novel method for high throughput production of mature hiPSC-derived neural tissues with defined structures that resemble those found in the SC.

Keywords: 3D bioprinting; neural tissue; motor neurons; pluripotent stem cells; biomaterials; spinal cord injury; lab on a printer; fibrin

\section{Introduction}

Spinal cord injury (SCI), a neurological disorder that disrupts communication within the central nervous system (CNS), can lead to impaired function [1]. The World Health Organization (WHO) reports 250,000-500,000 new cases annually, with the majority (90\%) being caused by traumatic lesions to the spinal cord (SC) [2]. The economic burden of SCI in Canadian patients ranges from $\$ 1.5$ million 
to $\$ 3$ million per patient, depending on the type of injury. These lesions can result from car crashes, violence-related wounds, and recreational activities [3]. The type of injury determines the effects on essential bodily functions performed by the motor, sensory, and autonomic systems, which can lead to movement impairment and deficiencies in the somatosensory system [3,4]. Furthermore, SCI increases the risk of developing diseases, such as multiple sclerosis [5].

Currently, no cure exists for SCI, so treatment methods focus on rehabilitation, reduction of pain and swelling, and pharmacological interventions. Cox et al. reported research on possible pharmacological treatments for different aspects of SCI in 2015. These treatments include Oxycyte, which provides oxygen to the injury site, reducing apoptosis [6]. Furthermore, the inhibition of the nuclear transcription factor, $\mathrm{NF}-\mathrm{K} \beta$, may reduce inflammation, and the antibiotic, Minocycline, has shown promising results in decreasing apoptosis in animal models [6]. The steroid, methylprednisolone, was used to treat acute SCI, but the side effects outweighed the benefits and its use has been discontinued [1]. One reason for the lack of effective treatments stems from the fact that the current tools for assessing promising drug targets for treating neurological disorders, like SCI, do not accurately predict toxicity and efficacy. Thus, the field of tissue engineering has been developing new techniques and methodologies that mimic the pathophysiology and functionality of the tissue found in the spinal cord.

Tissue engineering uses different combinations of cells, biomaterials, and biochemical factors to produce 3D tissues that resemble human physiology [7]. 3D models offer a significant advantage over 2D models. The 3D environments enable cells to develop into their correct morphology, resulting in more physiologically relevant function [8]. They also offer a microenvironment for encouraging cell-cell and cell-scaffold interactions, similar to how cells would develop normally in vivo [8]. Thus, tissue engineering allows us to study the cell function more precisely in vitro to determine how they would behave under the influence of certain drugs or varying physicochemical conditions.

Recent tissue engineering efforts have focused on the use of 3D bioprinting as a novel way to engineer tissues. 3D bioprinting produces 3D structures, often called constructs, using biocompatible materials known as bioinks where encapsulated cells can grow and proliferate [8]. Alternatives to 3D printing include freeze drying, gas foaming, mold casting, electrospinning, and heat compression to create a 3D structure [9]. Nevertheless, most of these methods cannot correctly mimic the necessary microarchitecture for stem cells to differentiate efficiently into neural phenotypes, as some of the aforementioned methods use toxic solvents that affect cell viability and function [9]. In order for the constructs to correctly mimic the SC tissue and serve as a preclinical model of SCI for drug screening applications, specific biological, chemical, and physical conditions must be met [8]. Bioprinting conditions enable the construction of favorable biochemical and mechanical environments, as well as the tunable construction of microstructures, generating an acceptable model to study SCI [9]. Nevertheless, it is important that during the printing protocol, cells remain undamaged. It has been reported that low cell viability can occur due to shear stress acting upon the cells during the printing process [8]. Parameters, such as pressure and temperature, may inflict changes on a cell's phenotype and thus, they should be carefully managed and their effects assessed [10].

Stem cells, such as human induced pluripotent stem cells (hiPSCs), can play a huge role in understanding SCI due to their ability to self-renew and to differentiate into any cell type in our body [11,12]. Scientists can design in vitro lab experiments that can directly translate into clinical studies by creating patient-specific tissues [13]. Differentiation protocols for hiPSCs need to ensure the development of a homogeneous neural cell population, as it has been reported that hiPSCs develop into neurons with less efficiency and more variability than embryonic stem cells (ESCs) [11,14]. Moreover, the use of hiPSC-derived neural progenitor cells (NPCs) limits differentiation to neuron-specific cell fates. Mothe et al. reported neuroregeneration and neuroprotection after NPCs transplant in rats with SCI [11]. 
Another important component to take into consideration for the generation of neural tissue is the bioink to be used for printing. For this study, a novel fibrin-based bioink was used to bioprint NPCs. The use of a fibrin-based bioink promotes neuronal differentiation and survival as it is biocompatible, human derived, contains cell adhesion signaling molecules, and allows the flow of nutrients [15]. In addition, this material will enable neural differentiation as it can mimic the biochemical and mechanical environment of the SC [8]. Being a naturally-derived material, fibrin has a decreased mechanical stability and a slow polymerization process $[8,15]$. The polymerization of fibrin starts with the addition of the enzyme, thrombin [16]. Alginate, which polymerizes quickly, can be mixed with fibrin as a second component and make the bioink suitable for bioprinting [8]. To address the challenge of the lack of mechanical stability of fibrin scaffolds, small molecules, such as genipin, can enhance the structural properties of the scaffold and promote neural outgrowth [16]. The cationic polysaccharide, chitosan, is the third component of the novel bioink used to provide mechanical strength to the scaffolds by ionically interacting with alginate and covalently crosslinking with genipin [17].

Inkjet, extrusion, laser-assisted, and stereolithography are the main strategies for bioprinting tissues $[18,19]$. However, one of the major challenges faced when using these technologies is the amount of shear stress the cells receive as they are extruded, which leads to low cell viability and lack of long term-functionality of the tissues [20]. The Lab-on-a-Printer ${ }^{\mathrm{TM}}$ (LOP) technology integrates microfluidic channels with printing technology that allows material processing prior to printing and programmable parameters to obtain specific compositions in the printed materials [21]. In this study, the RX1 ${ }^{\mathrm{TM}}$ Bioprinter from Aspect Biosystems ${ }^{\mathrm{TM}}$ was used to bioprint NPCs. This bioprinter offers the possibility of printing 3D scaffolds with cell-laden bioinks where programmable patterns can be created [22]. Speed and fiber composition are regulated while printing on a layer-by-layer basis. The RX1 ${ }^{\mathrm{TM}}$ uses a LOP ${ }^{\mathrm{TM}}$ microfluidic printhead that prints complex and heterogeneous structures with different cell types and materials in a reproducible manner [22]. These characteristics lead to the bioprinting of a precise design with specific micro and macro architectures while the bioink components are evenly distributed in the 3D structure. In the case of SC, it is necessary to mimic precisely the anatomical and physiological characteristics in order to develop a correct model of the disease to perform drug screening. Tuning the aforementioned characteristics will enable the differentiation of NPCs into motor neurons (MNs), one of the main type of neurons affected during SCI and whose functionality needs to be re-established [23].

The objective of this study was to bioprint hiPSC-derived neural tissues that matured into MNs in combination with a novel fibrin-based bioink using the $\mathrm{RX} 1^{\mathrm{TM}}$ bioprinter in rapid manner. Here, we analyze the composition of 30-day cultures of bioprinted hiPSC-derived NPCs exposed to the small molecules, CHIR99021 (CHIR), SB431542 (SB), LDN-193189 (LDN), purmorphamine (Puro), and retinoic acid (RA), to promote MN differentiation. Previous studies have demonstrated that the small molecules, SB431542 and DMH1, induce dual-SMAD inhibition, a requirement for neural pattering in stem cells [24,25]. The molecule, LDN-193189, is also a BMP inhibitor and can be used instead of DMH1 [24]. Moreover, the small molecule, CHIR99021, activates the Wnt pathway, enhancing neural patterning of pluripotent stem cells [25]. In addition, the combination of RA and puro promote pluripotent stem cell differentiation into motor neurons [25-27]. Cylindrical constructs were printed using the $\mathrm{RX} 1^{\mathrm{TM}}$ bioprinter with seven layers and a $1 \mathrm{~cm}$ diameter in a process that took under $5 \mathrm{~min}$ to generate four constructs. Cell viability, immunocytochemistry (ICC), and flow cytometry studies were performed to analyze the bioprinted constructs and their differentiation into MNs. 


\section{Materials and Methods}

\subsection{Culture and Expansion of NPCS}

hiPSC-derived NPCs were obtained as previously described from the hiPSC line, 1-DL-01, from WiCell $[16,28]$. Experiments using hiPSC-derived NPCs were conducted with the approval of the University of Victoria's Human Ethics Committee under protocol number: 12-187. Briefly, NPCs were cultured in STEMdiff ${ }^{\mathrm{TM}}$ Neural Progenitor Medium (NPM) (05834, Stemcell Technologies, Vancouver, BC, Canada) containing 1 X STEMdiff ${ }^{\mathrm{TM}}$ Neural Progenitor Supplement A (05836, Stemcell Technologies, Vancouver, BC, Canada), 1 X STEMdiff ${ }^{\mathrm{TM}}$ Neural Progenitor Supplement B (05837, Stemcell Technologies Vancouver, BC, Canada), and 1\% Penicillin-Streptomycin (Penstrep) (P4333, Sigma, St. Louis, MO, USA) in 6-well cell culture plates coated with laminin (L2020, Sigma, St. Louis, MO, USA) and poly-L-ornithine (PLO) (P4957, Sigma, St. Louis, MO, USA). The cultured NPCs were maintained at $37{ }^{\circ} \mathrm{C}$ with $5 \% \mathrm{CO}_{2}$ and media change was performed on a daily basis. Once the cultures reached $80 \%$ confluence the cells were cryopreserved in liquid nitrogen.

\subsection{Bioprinting Process}

NPCs were thawed and resuspended in the bioink solution composed of $20 \mathrm{mg} / \mathrm{mL}$ of fibrinogen (341578, Sigma, St. Louis, MO, USA), $0.5 \%$ w/v of alginate $(120,000-190,000 \mathrm{~g} / \mathrm{mol}, \mathrm{M} / \mathrm{G}$ ratio 1.56$)$ (180947, Sigma, St. Louis, MO, USA), and $0.3 \mathrm{mg} / \mathrm{mL}$ of Genipin dissolved in de-ionized water (G4796, Sigma, St. Louis, MO, USA) in tris-buffered saline). The conical tube containing the bioink and NPCs was coupled to the Material 1 channel of the LOP ${ }^{\mathrm{TM}}$ printhead (Aspect Biosystems, Vancouver, BC, Canada). The crosslinking solution contained $20 \mathrm{mg} / \mathrm{mL}$ of calcium chloride (449709, Sigma, St. Louis, MO, USA), $0.075 \% w / v$ of chitosan (C3646, Sigma, St. Louis, MO, USA), and $1.7 \mathrm{U} / \mathrm{mL}$ of thrombin (T7009-1KU, Sigma, St. Louis, MO, USA). Cylindrical constructs of $1 \mathrm{~cm}$ diameter were bioprinted using the RX1 bioprinter (Aspect Biosystems, Vancouver, BC, Canada) with 7 layers of $\sim 176 \mu \mathrm{m}$ diameter. The cross-linking process takes a few seconds as it is initiated inside the printhead at the intersection point between the bioink and crosslinker channels shown in Figure 1. Specified pressures are applied to each channel, controlling the flow rate to allow enough time for the crosslinking reaction. Fibers coming out of the printhead are crosslinked before they are deposited. The printing speed was $20 \mathrm{~mm} / \mathrm{s}$ and channel pressures consisted of 20 mbar for the bioink, 40 mbar for the crosslinker, and 100 mbar for buffer solution. Four structures were bioprinted simultaneously in a layer-by-layer fashion in under $5 \mathrm{~min}$. Figure 1a shows a schematic representation of the LOP ${ }^{\mathrm{TM}}$ printhead. Figure $1 \mathrm{~b}$ shows the bioprinted cylindrical construct. All the procedures were performed under sterile conditions. After bioprinting, the constructs were gently transferred to culture plates coated with laminin and PLO. $1.5 \mathrm{~mL}$ of NPM was added to each well and these cultures were incubated at $37^{\circ} \mathrm{C}$ with $5 \% \mathrm{CO}_{2}$. Measurements of fiber diameters were performed using the software, Image J, after imaging with a Leica DMI3000B (Leica Biosystems, Wetzlar, Germany) microscope and QImaging RETIGA 2000R camera (QImaging, Surrey, BC, Canada) at 100 $\times$ magnification. Imaging and diameter measurement of the bioprinted construct was performed using Cytation $5^{\mathrm{TM}}$ and the software, Gen5 version 3.05 (BioTek instruments, Winooski, VT, USA). 


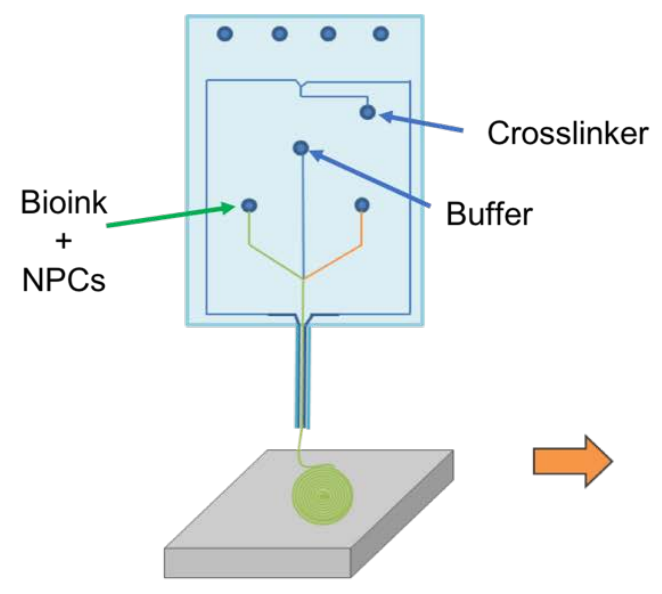

(a)

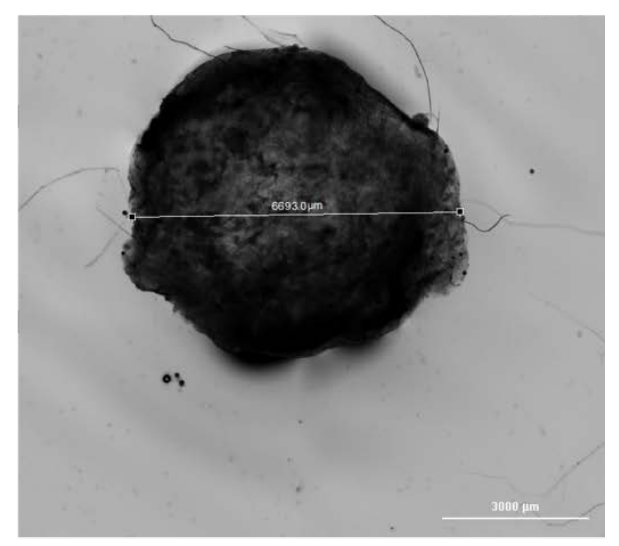

(b)

Figure 1. (a) Schematic representation of $\mathrm{LOP}^{\mathrm{TM}}$ printhead and bioprinted cylindrical construct; (b) bioprinted cylindrical construct consisting of hiPSC-derived NPCs and our novel fibrin-based bioink. Scale bar represents $3000 \mu \mathrm{m}$. hiPSC: human induced pluripotent stem cells; NPCs: neural progenitor cells.

\subsection{Culture of Bioprinted Constructs}

The printed constructs were treated with a cocktail of small molecules suspended in NPM containing $1 \%$ Penicillin-Streptomycin to promote differentiation of the bioprinted NPCs into MNs. Media changes were performed every third day by replacing $750 \mu \mathrm{L}$ with fresh media from day 1 to day 15. Table 1 shows the five different treatments analyzed with the combinations of small molecules added, each treatment is represented by a letter. Treatments consisted of: (i) Negative (N+); $2 \mu \mathrm{M}$ of SB, $2 \mu \mathrm{M}$ of LDN and $1 \mu \mathrm{M}$ of CHIR; (ii) uro positive (P+); $0.1 \mu \mathrm{M}$ of Puro, $2 \mu \mathrm{M}$ of SB, $2 \mu \mathrm{M}$ of LDN and $1 \mu \mathrm{M}$ of CHIR; (iii) RA positive (R+); $0.5 \mu \mathrm{M}$ of RA, $2 \mu \mathrm{M}$ of SB, $2 \mu \mathrm{M}$ of LDN and $1 \mu \mathrm{M}$ of CHIR; (iv) Puro and RA positive (PR+); $0.1 \mu \mathrm{M}$ of Puro, $0.5 \mu \mathrm{M}$ of RA, $2 \mu \mathrm{M}$ of SB, $2 \mu \mathrm{M}$ of LDN and $1 \mu \mathrm{M}$ of CHIR; (v) Puro and RA negative (PR-); $0.1 \mu \mathrm{M}$ of Puro and $0.5 \mu \mathrm{M}$ of RA. Starting on day 15 , concentrations of puro and RA were $0.1 \mu \mathrm{M}$ and $0.5 \mu \mathrm{M}$, respectively. By day 15 , the media was gradually replaced as previously reported by BrainPhys Neuronal Medium (BP) (05790, Stemcell, Vancouver, CA) supplemented with growth factors; $10 \mathrm{ng} / \mathrm{mL}$ of GDNF $10 \mathrm{ng} / \mathrm{m}$ (450-10, Peprotech, Rocky Hill, NJ, USA), $10 \mathrm{ng} / \mathrm{mL}$ of IGF-1 (AF-100-11, Peprotech, Rocky Hill, NJ, USA), $1 \mu$ M of C-AMP (A6885, Sigma, St. Louis, MO, USA), 1\% of N2 (17502048 Thermo Fisher, Waltham, MA, USA), 1\% of B27 (17504001, Thermo Fisher, Waltham, MA, USA), $200 \mathrm{ng} / \mathrm{mL}$ of Ascorbic Acid (Sigma, St. Louis, MO, USA), $10 \mathrm{ng} / \mathrm{mL}$ of BDNF (450-02, Peprotech, Rocky Hill, NJ, USA ), and 1\% penstrep [27]. The bioprinted constructs were maintained at $37^{\circ} \mathrm{C}$ with $5 \% \mathrm{CO}_{2}$

Table 1. Name, letter code, and treatment for each group.

\begin{tabular}{ccc}
\hline Name & Letter Code & Treatment \\
\hline Negative & $\mathrm{N}+$ & CHIR, SB, LDN \\
Puro & $\mathrm{P}+$ & Puro + CHIR, SB, LDN \\
RA & $\mathrm{R}+$ & RA + CHIR, SB, LDN \\
Puro and RA & $\mathrm{PR}+$ & Puro and RA + CHIR, SB, LDN \\
Puro and RA & PR- & Puro and RA \\
\hline
\end{tabular}




\subsection{Cell Viability}

\subsubsection{Viability Assay}

On day 7 , the constructs $(n=3)$ were degraded by removing culture media followed by incubation with a solution of $25 \mathrm{mM}$ of Sodium Citrate (Sigma, St. Louis, MO, USA). The constructs were then placed on a shaker at $25 \mathrm{rpm}$ at $37^{\circ} \mathrm{C}$. Once most of the bioink was degraded, $0.125 \%$ of trypsin-EDTA (Fisher Scientific, Pittsburgh, PA, USA) was added for $5 \mathrm{~min}$ followed by the addition of fetal bovine serum (FBS) (10438018, Thermo Fisher, Waltham, MA, USA). Each treatment was placed on a $15 \mathrm{~mL}$ conical tube and centrifuged at $300 \mathrm{rpm}$ for $5 \mathrm{~min}$. Preparation of the cell suspension was performed as previously reported following the Guava ViaCount ${ }^{\circledR}$ protocol for cell viability determinations [16]. The supernatant was removed, and the pellet was resuspended in $1 \mathrm{~mL}$ of phosphate buffered solution (PBS) (10010023, Thermo Fisher, Waltham, MA, USA). $20 \mu \mathrm{L}$ of the cell suspension was mixed with $380 \mu \mathrm{L}$ of Guava ViaCount reagent ${ }^{\circledR}$ (4000-0040, Millipore, Burlington, MA, USA). $100 \mu \mathrm{L}$ were placed on each of the 96-well plate and cell viability was determined using the Guava EasyCyte HT (Millipore, Burlington, MA, USA) flow cytometer. Statistical analysis was done using one-way ANOVA followed by Tukey's post-hoc analysis using GraphPad prism 6 statistics software. Confidence levels were 95\% $(p<0.05)$.

\subsubsection{Flow Cytometry}

Cell marker expression of the early neuronal markers, ßeta tubulin-III ( $\beta$ T-III) ((IC1195C R\&D), oligodendrocyte transcription factor 2 (Olig2) (IC2230P R\&D systems, Minneapolis, MN, USA), and the protein homeobox 9 (HB9) (bs-11320R, Bioss antibodies), was quantified on day 15. The bioprinted constructs were degraded as previously mentioned using a solution of $25 \mathrm{mM}$ of sodium citrate, $0.125 \%$ of trypsin-EDTA, and FBS. The resulted cell suspension was processed as previously reported [27,29]. Briefly, the cell suspension was washed three times with PBS by centrifuging at $300 \mathrm{~g}$ for $5 \mathrm{~min}$. The cell suspension was then fixed and stained per the manufacturer's instructions (R\&D systems, Minneapolis, $\mathrm{MN}$, USA). Isotype controls consisted of mouse IgG2A PerCP-conjugated Isotype control (IC003C R\&D systems, Minneapolis, MN, USA ) and normal mouse IgM PE-conjugated Control (IC015P R\&D systems, Minneapolis, MN, USA). The analysis was performed using the Guava EasyCyte HT flow cytometer (Millipore, Burlington, MA, USA). Statistical analysis was done using one-way ANOVA followed by Tukey's post-hoc analysis using GraphPad prism 6 statistics software. Confidence levels were $95 \%(p<0.05)$ and $99.9 \%(p<0.001)$.

\subsection{Immunocytochemistry}

Immunohistochemical analysis of protein expression was performed on day 30 for $\beta$ T-III, choline acetyl transferase (ChaT), and glial fibrillary acidic protein (GFAP). The constructs were collected and fixed for $1 \mathrm{~h}$ in $4 \%$ paraformaldehyde (PFA) (AC416785000, Acros Organics, Geel, Belgium) in PBS solution. Three washes were performed by adding fresh PBS and agitating at $25 \mathrm{rpm}$ at $4{ }^{\circ} \mathrm{C}$. The cells were permeabilized by adding $0.1 \%$ of Triton-X (HT501128, Sigma, St. Louis, MO, USA) in PBS for $45 \mathrm{~min}$ under agitation at $25 \mathrm{rpm}$ at $4{ }^{\circ} \mathrm{C}$. Blocking was done using $5 \%$ Normal Goat Serum (NGS) (NS02L, Sigma, St. Louis, MO, USA) in PBS for $2 \mathrm{~h}$ under the same conditions. Primary antibodies were incubated overnight under the same conditions at the concentrations of 1:500 for mouse anti-TUJ1 (60052, Stemcell, Vancouver, BC, Canada), 1:1000 rabbit anti-HB9 (ABN174, Millipore, Burlington, MA, USA), and 1:50 goat anti-ChAT (ab144p abcam). Following three washes with PBS, the constructs were incubated for $4 \mathrm{~h}$ under agitation at $25 \mathrm{rpms}$ and room temperature with the secondary antibodies at the concentrations of 1:500 for Alex Fluor Donkey anti-goat (405) (Ab175664 abcam, Eugene, OR, USA), 1:500 AlexaFluor 568 Donkey Anti-Mouse (Ab175700 abcam, Eugene, OR, USA), and 1:500 AlexaFluor 488 Donkey Anti-Rabbit (Ab150073, abcam, Eugene, OR, USA). 


\section{Results}

\subsection{Bioprinted NPCS in Cylindrical Constructs}

The bioprinted constructs were printed in a layer-by-layer fashion. The structures consisted of $1 \mathrm{~cm}$ diameter- cylinders with seven layers of fibers with an average diameter of $176 \pm 16 \mu \mathrm{m}$ and the total construct height was $\sim 1.2 \mathrm{~mm}$. The NPCs were homogeneously placed throughout the fibers within the constructs as shown in Figure 2 during days 0 to 10. The bioink provided mechanical and biochemical support for the cells and slowly degraded over time. By day 30, most of the bioink had degraded while the remaining cellular structures remained attached to the tissue culture well.

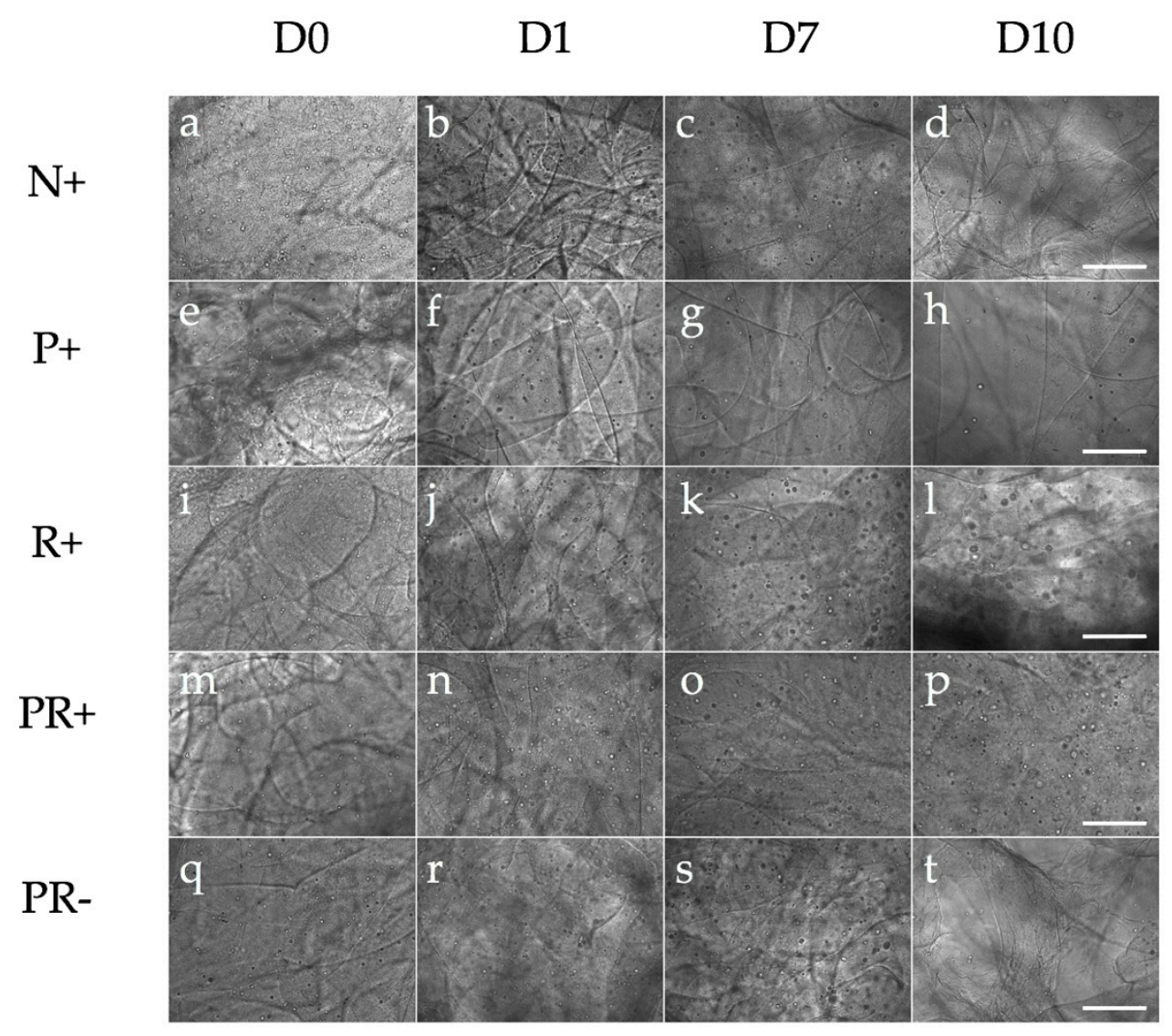

Figure 2. Phase contrast images of bioprinted constructs on days $0,1,7$, and 10 (a-d). Negative group (N with small molecules); (e-h) Puro (P+ with small molecules); (i-l) RA (RA+ with small molecules), $(\mathbf{m}-\mathbf{p})$ Puro and RA (PR+ with small molecules); (q-t) Puro and RA (PR- no small molecules). Scale bar represents $300 \mu \mathrm{m}$.

\subsection{Cell Viability}

Quantitative analysis for cell viability was performed at day 7 using ViaCount assay from Millipore ( $\mathrm{n}=3$ for all groups) (Figure 3$)$. On day 7 , cell viability for $\mathrm{N}+$ was $(89.2 \pm 8.9 \%), \mathrm{P}+(93.3 \pm 5.1 \%)$, $\mathrm{R}+(90.9 \pm 6.3 \%), \mathrm{PR}+(87.3 \pm 0.8 \%)$, and $\mathrm{PR}-(81.2 \pm 1.8 \%)$. No statistically significant differences were observed. 


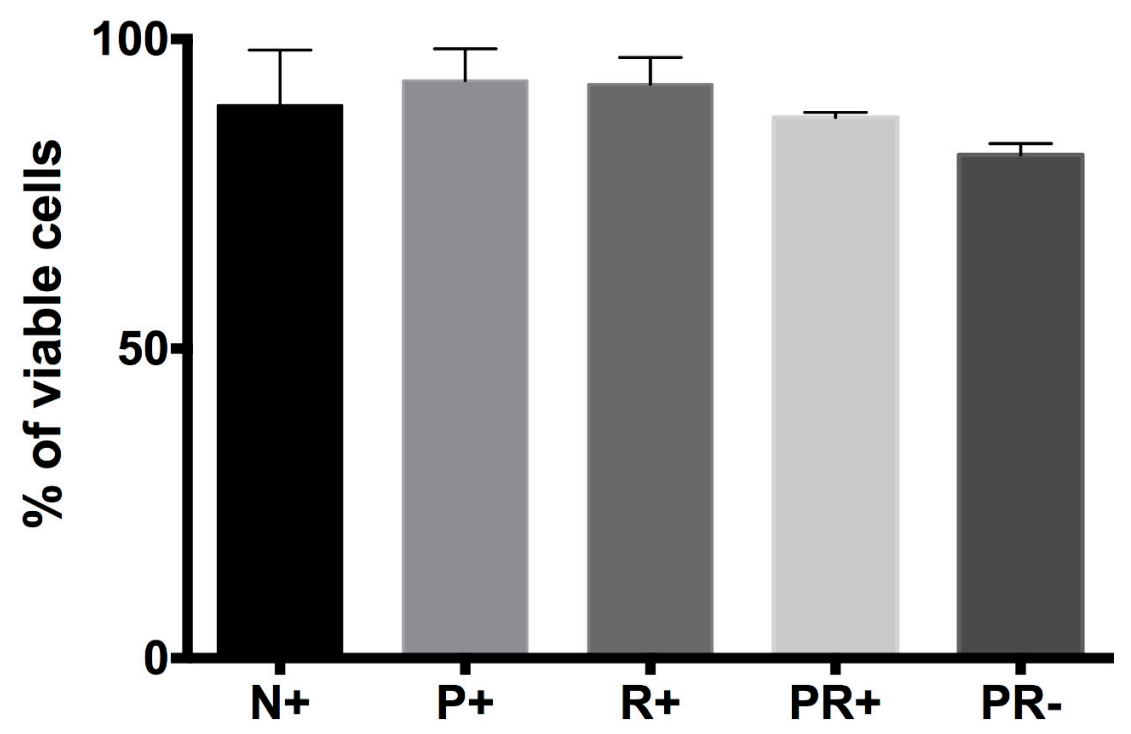

Figure 3. Cell viability of the NPCs for all groups on day 7 after being bioprinted. $n=3$ for all groups. Data is reported as the mean with the error bars representing the standard deviation. One-way ANOVA and Tukey post-hoc analysis was performed for statistical analysis using a confidence level of $95 \%(p<0.05)$. No statistically significant differences were observed. The same data visualized as a scatterplot can be seen in Supplementary Figure S1.

\subsection{Protein Expression of 3D Bioprinted Neural Tissues}

\subsubsection{Flow Cytometry}

On day 15, flow cytometry was performed to quantify cell marker expression of $\beta$ T-III, Olig2, and HB9 (mature MN) (Figure 4). Expression of $\beta$ T-III was the highest for $\mathrm{P}+(45 \pm 20.9 \%)$, followed by $\mathrm{PR}+(33.1 \pm 13.4 \%), \mathrm{N}+(21.3 \pm 21.3 \%)$ and $\mathrm{PR}-(4.3 \pm 1.6 \%)$. No statistically significant differences were observed for $\beta$ T-III expression. Olig2 expression was the highest for $\mathrm{N}+(68.8 \pm 6.9 \%)$, followed by $\mathrm{PR}-(61.1 \pm 9.9 \%)$ and $\mathrm{R}+(46 \pm 4.8 \%)$. The lowest level of expression was observed in PR+ $(3.16 \pm 1.10 \%)$. Statistically significant differences were observed between $\mathrm{N}+$ and $\mathrm{PR}+, \mathrm{R}+$ and $\mathrm{PR}+$, and PR+ and PR-. HB9 expression was highly expressed for all groups at this time point. R+ showed the highest level of expression $(99.6 \pm 0.4 \%)$, whereas PR+ and PR- had lower and similar levels of HB9 expression, $(76.7 \pm 10.8 \%)$ and $(75.5 \pm 10.3 \%)$, respectively. $\mathrm{N}+$ showed the lowest level of expression for HB9 $(61.1 \pm 5 \%)$. Statistically significant differences were observed for $\mathrm{N}+$ and $\mathrm{R}+$.

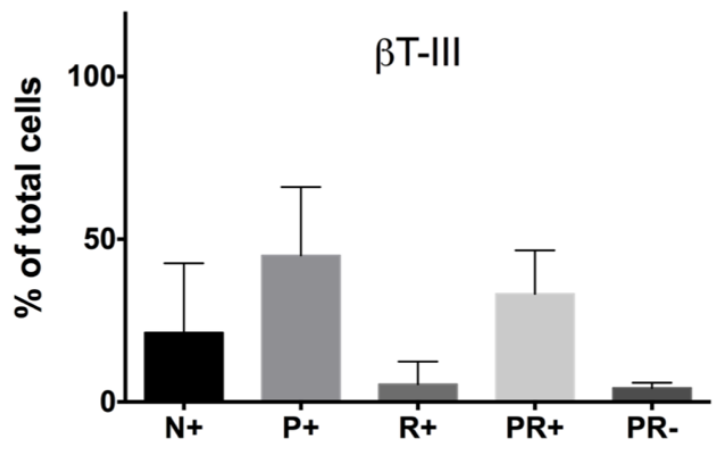

(a)

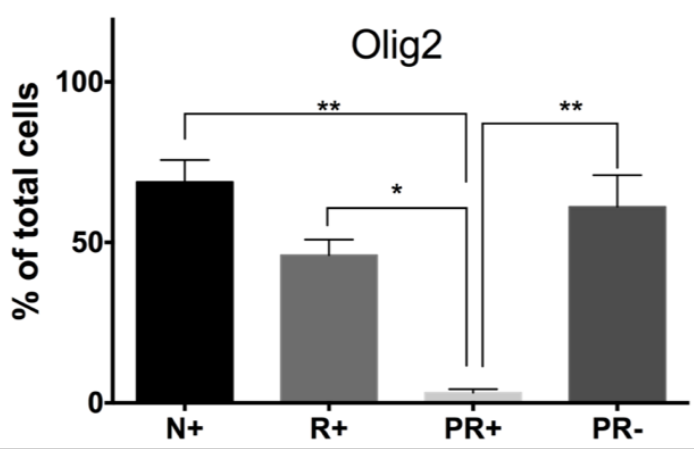

(b)

Figure 4. Cont. 


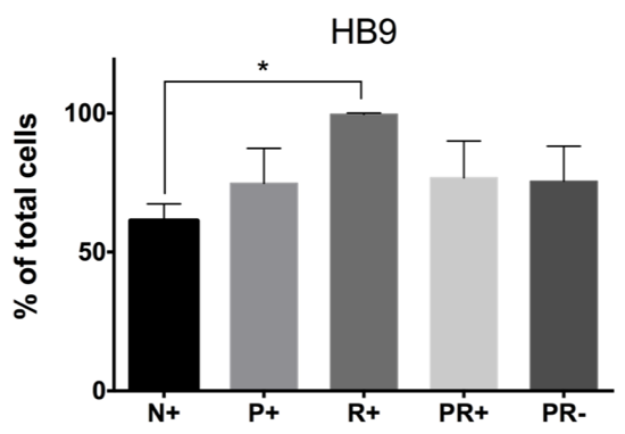

(c)

Figure 4. Flow cytometry of 3D bioprinted NPCs after 15 days of culture in vitro (a) $\beta$ T-III; (b) Olig2; (c) HB9. $\mathrm{n}=3$ for all groups. One-way ANOVA and Tukey post-hoc analysis was performed for statistical analysis using a confidence level of $95 \%(p<0.05)$ and $99.9 \%(p<0.001)$. ${ }^{*}$ represents $p<0.05$ and ${ }^{* *} 99.9 \%$ $p<0.001$. The same data visualized as a scatterplot can be seen in Supplementary Figure S2.

\subsubsection{Immunocytochemistry}

ICC was performed at day 30 for all groups for $\beta$ T-III, ChaT, and GFAP (Figure 5). $\beta$ T-III expression was positive for all groups. ChaT expression was primarily expressed in $\mathrm{PR}+$, however, $\mathrm{N}$ and PR - also showed expression of ChaT. GFAP expression was positive for $\mathrm{N}, \mathrm{R}+$, and PR-.
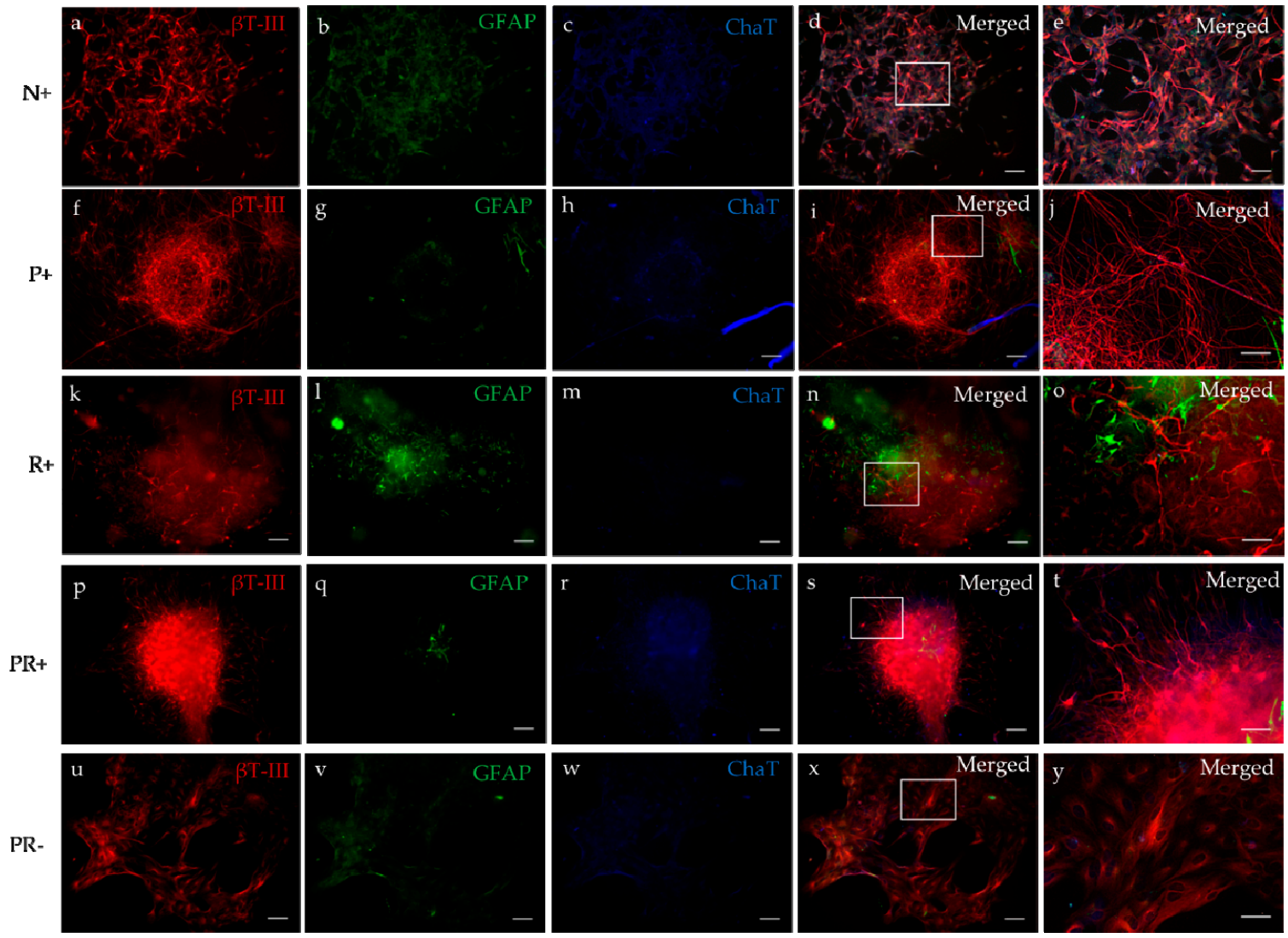

Figure 5. Immunocytochemistry after 30 days of culture in vitro. $(\mathbf{a}-\mathbf{e}) \mathrm{N}+;(\mathbf{f}-\mathbf{j}) \mathrm{P}+;(\mathbf{k}-\mathbf{o}) \mathrm{R}+$; (p-t) $\mathrm{PR}+$; $(\mathbf{u}-\mathbf{y})$ PR-. Scale bar for $(\mathbf{a}-\mathbf{d}, \mathbf{f}-\mathbf{i}, \mathbf{k}-\mathbf{n}, \mathbf{p}-\mathbf{s}, \mathbf{u}-\mathbf{x})$ represents $100 \mu \mathrm{m}$. Scale bar for $(\mathbf{e}, \mathbf{j}, \mathbf{o}, \mathbf{t}, \mathbf{y})$ represent $50 \mu \mathrm{m}$.

\section{Discussion}

Previous studies have extensively explored the use of a 3D environment to create neural tissue by using natural or synthetic polymers. Such strategies are focused on the creation of hydrogel 
constructs, nerve guidance channels, or electrospinning [16,30-32]. However, those approaches require a large amount of labor and raw materials while their reproducibility remains challenging. Bioprinting technologies offer great advantages over conventional tissue engineering methods as they require less labor and materials and can be automated, producing high-throughput and reproducible processes $[18,19]$. In addition, patterned-specific structures with micro-architectures at the micrometer size resolution can be created to mimic the native tissues $[18,19]$. Recent studies have used 3D bioprinting technologies where multichannel scaffolds were 3D printed and NPCs within a bioink were extruded in a layer-by-layer fashion [33]. A different study performed by Gu et al. created functional mini tissues by bioprinting human neural stem cells (hNSCs) using a 3D-bioplotted system where the bionk is extruded, followed by incubation in crosslinking solution [34].

In this study, we successfully bioprinted NPCs using the microfluidic LOP ${ }^{\mathrm{TM}}$ and differentiated these cells into ChaT expressing MNs after 30 days. With this LOP ${ }^{\mathrm{TM}}$ technology, all the components of the bioink are mixed prior to the printing process and crosslinked at the $200 \mu \mathrm{m}$ " $\mathrm{Y}$ " junction prior to ejection from the single nozzle. This special feature, where the bioink is surrounded by two crosslinking channels at the $\mathrm{Y}$ junction, initiates the crosslinking process from both sides and creates a sheath surrounding the bioink that protects the cells from shear-stress while being extruded. In addition, the $700 \mu \mathrm{m}$ extrusion nozzle contained in this LOPTM is made out of PDMS and is therefore flexible and less stiff in comparison with other expression needles used in other studies [33-35]. Using a pneumatic pressure driven flow, our fibers are extruded at $25 \mathrm{mbar}$ for the bioink, and an increased pressure of $40 \mathrm{mbar}$ for the crosslinker, leading to the creation of printed fiber diameters of $(176 \pm 16 \mu \mathrm{m})$, with all the bioink components homogenously distributed. These diameters are similar to those reported previously when bioprinting NPCs using a point dispensing printing method [33].

On day 7 , cell viability was above $81 \%$ for all groups, where $\mathrm{P}+$ and $\mathrm{R}+$ showed the highest levels of cell viability at $93 \%$ and $91 \%$, respectively. These percentages are higher than those reported by Joung et al., where cell viability is reported to be $>75 \%$ after four days of printing iPSC-derived spinal neural progenitor cells (sNPCs) and oligodendrocyte progenitor cells (OPCs) in a 50\% Matrigel bioink [33]. Flow cytometry analysis was performed to assess the differentiation of the bioprinted NPCs cultured with combinations of small molecules that have shown to promote $\mathrm{MN}$ differentiation after 15 days of culture $[25,26]$. At this time point, $\mathrm{P}+$ and $\mathrm{PR}+$ showed the highest levels of the neuronal marker, $\beta$ T-III, expression, with no statistically significant differences between groups. Expression of the oligodendrocyte transcription factor 2 (Olig2) was also quantified at this time point, and the overall expression for most groups was higher in comparison, with $\beta \mathrm{T}-\mathrm{III}$. $\mathrm{N}+$ and PR+ showing the highest levels of expression ( $>60 \%$ ), suggesting that either combination of small molecules (CHIR, SB, and LDN for $\mathrm{N}+$ ) or (puro and RA for PR-) have a similar impact on the differentiation of NPCs into Olig2+ cells. These levels of $\beta$ T-III and Olig2 expression are significantly higher than that reported by Gu et al., where hiPSC-derived NPCs were bioprinted and differentiated, with a maximum gene expression of $\beta$ T-III of $~ 2 \%$ and $15 \%$ for Olig2 after three weeks of differentiation [34].

The expression of the mature MN marker, HB9, at this time point was highly expressed for all groups at $>61 \%$. The highest level of HB9 expression was observed in the R+ group, however, the only statistically significant differences were observed between $\mathrm{N}+$ and $\mathrm{R}+$. Interestingly, P+, PR+, and PRshowed very similar levels of expression, suggesting that the presence of puro, alone or in combination with RA, is crucial for differentiating HB9+ neurons from NPCs using our novel bioink. However, given that PR+ and PR- have similar levels of HB9 expression with a yield of $\sim 76 \%$, either treatment could be used for future analysis. However, $\mathrm{R}+$ with the combination or RA and CHIR, SB, LDN is the most efficient, showing 99\% yield of HB9+ MNs after 15 days.

Our 3D bioprinted tissues were cultured for up to 30 days in vitro for further maturation, and characterization of MNs compared with previous studies where ICC staining was performed after seven days of culture [33]. Nonetheless, $\mathrm{Gu}$ et al. cultured their bioprinted constructs for up to 40 days, showing the expression of mature neural markers, such as microtubule associated protein 2 (MAP2), gamma-aminobutyric acid GABA), and Synaptophysin [35]. ICC was performed for $\beta$ T-III, 
the astrocyte marker (GFAP), and choline acetyl transferase (ChaT) to assess neuronal and glial cell differentiation. All of the groups showed $\beta$ T-III expression; interestingly, $\mathrm{P}+$ and $\mathrm{PR}+$ self-aggregated into a spheroid structure as previously observed [35]. At this time point, all the scaffolds had been degraded, demonstrating that our bioink supported the cellular secretion of the required extra cellular matrix (ECM) components to maintain a 3D structure. GFAP expression was positive for $\mathrm{N}, \mathrm{R}+, \mathrm{PR}+$, and $\mathrm{PR}-$. ChaT expression was positive for $\mathrm{N}+, \mathrm{PR}-$, and $\mathrm{PR}+$. Interestingly, $\mathrm{PR}+$ showed the most cell aggregation and expression of $\beta \mathrm{T}$-III and ChaT, and the lowest expression for GFAP, suggesting that the combination of these small molecules provides a better environment for $\mathrm{MN}$ differentiation and maturation.

Further maturation and characterization of the tissues will be necessary in order to fully characterize their maturity and electrophysiological characteristics. These bioprinted neural tissues can be used as a complementary pre-clinical model to assess drug safety and toxicity in the early development of clinical trials. hiPSCs can be derived from the patient and matured into neural progenitor cells in order to perform such an analysis with patient specific cells. In the future, drug releasing microspheres can be deposited along with the cell-suspension within the bioink, where each fiber contains the required drug-releasing microspheres to promote differentiation into different cell types. More than one type of cell can be differentiated within these bioprinted tissues in order to mimic a more defined micro-architecture of the SC tissue along with a blood spinal cord barrier (BSCB).

\section{Conclusions}

Here, we use a novel bioprinting method for generating hiPSC-derived neural tissues similar to the tissue found in the SC as indicated by their survival and expression of the MN markers, Olig2, HB9, and ChaT. Such bioprinted neural tissues can potentially serve as a tool for screening potential drugs for treating SCI, as well as give insight into developing potential cell therapies for treating SCI.

\section{Patents}

A provisional patent has been filed with regards to our novel bioink.

Supplementary Materials: The following are available online at http:/ / www.mdpi.com/2076-3417/8/12/2414/s1. Figure S1: Scatter plot representation of cell viability of the NPCs for all groups on day 7 after being bioprinted. $n=3$ for all groups. Data is reported as the mean with the error bars representing the standard deviation. One-way ANOVA and Tukey post-hoc analysis was performed for statistical analysis using a confidence level of $95 \%(p<0.05)$. No statistically significant differences were observed ; Figure S2: Flow cytometry of 3D bioprinted NPCs after 15 days of culture in vitro (a) $\beta \mathrm{t}$-III; (b) Olig2; (c) HB9. $\mathrm{n}=3$ for all groups. One-way ANOVA and Tukey post-hoc analysis was performed for statistical analysis using a confidence level of $95 \%(p<0.05)$ and $99.9 \%(p<0.001)$. ${ }^{*}$ represents $p<0.05$ and $* * 99.9 \% p<0.001$.

Author Contributions: L.d.l.V. designed, set-up, performed, and supervised experimental procedures and data analysis. Wrote, reviewed, and edited manuscript. E.A. and L.A. developed methodology, assisted on experimental set-up, and helped addressing reviews. D.A.R.G. and V.A.d.S. assisted on experimental set-up, data analysis, and writing of the initial manuscript. S.M.W. provided input into experimental design, provided feedback and supervision on the experimental analysis, as well as editing on the manuscript and addressing reviews.

Funding: This work was funded by the British Columbia Innovation Council's Ignite award, the NSERC Discovery Grants program, and the Canada Research Chairs programs.

Acknowledgments: We also thank Simon Beyer and Samuel Wadsworth from Aspect Biosystems for their technical support of this research project.

Conflicts of Interest: Willerth has a collaborative research agreement with Aspect Biosystems to commercialize the results of their BCIC Ignite award.

\section{References}

1. Maitin, I.B.; Cruz, E. Current Diagnosis E Treatment: Physical Medicine \& Rehabilitation; McGraw-Hill Education: New York, NY, USA, 2015.

2. Spinal Cord Injury. Available online: http://www.who.int/news-room/fact-sheets/detail/spinal-cordinjury (accessed on 2 November 2018). 
3. Fehlings, M.G.; Vaccaro, A.R.; Ditunno, J.; Vaccaro, A.R.; Rossignol, S.; Burns, A. Essentials of Spinal Cord Injury: Basic Research to Clinical Practice; Thieme Medical Publishers, Incorporated: New York, NY, USA, 2012.

4. Pal, R.; Singh, M.; Kumar, K.; Jahanavi, V.M.; Jena, R.; Munivenkatappa, A.; Bhandarkar, P.; Agrawal, A. Understanding the Presentations and Patterns of Traumatic Spinal Cord Injuries to Develop the Data Collection Format. Indian J. Neurotrauma 2017, 14, 135-141. [CrossRef]

5. Lin, C.-W.; Huang, Y.-P.; Pan, S.-L. Spinal Cord Injury Is Related to an Increased Risk of Multiple Sclerosis: A Population-Based, Propensity Score-Matched, Longitudinal Follow-Up Study. J. Neurotrauma 2015, 32, 655-659. [CrossRef] [PubMed]

6. Cox, A.; Varma, A.; Banik, N. Recent Advances in the Pharmacologic Treatment of Spinal Cord Injury. Metab. Brain Dis. 2015, 30, 473-482. [CrossRef] [PubMed]

7. Temenoff, J.S.; Mikos, A.G. Biomaterials: The Intersection of Biology and Materials Science; Pearson/Prentice Hall: Upper Saddle River, NJ, USA, 2008; p. 1.

8. Thomas, M.; Willerth, S.M. 3-D Bioprinting of Neural Tissue for Applications in Cell Therapy and Drug Screening. Front. Bioeng. Biotechnol. 2017, 5, 69. [CrossRef] [PubMed]

9. Wang, M.; Zhai, P.; Chen, X.; Schreyer, D.J.; Sun, X.; Cui, F. Bioengineered Scaffolds for Spinal Cord Repair. Tissue Eng. Part B Rev. 2011, 17, 177-194. [CrossRef] [PubMed]

10. Gao, G.; Cui, X. Three-dimensional bioprinting in tissue engineering and regenerative medicine. Biotechnol. Lett. 2016, 38, 203-211. [CrossRef] [PubMed]

11. Mothe, A.J.; Tator, C.H. Advances in stem cell therapy for spinal cord injury. J. Clin. Investig. 2012, 122, 3824-3834. [CrossRef] [PubMed]

12. Nandoe Tewarie, R.S.; Hurtado, A.; Bartels, R.H.; Grotenhuis, A.; Oudega, M. Stem Cell-Based Therapies for Spinal Cord Injury. J. Spinal Cord Med. 2009, 32, 105-114. [CrossRef] [PubMed]

13. Shi, Y. Induced pluripotent stem cells, new tools for drug discovery and new hope for stem cell therapies. Curr. Mol. Pharmacol. 2009, 2, 15-18. [CrossRef] [PubMed]

14. Hu, B.-Y.; Weick, J.P.; Yu, J.; Ma, L.-X.; Zhang, X.-Q.; Thomson, J.A.; Zhang, S.-C. Neural differentiation of human induced pluripotent stem cells follows developmental principles but with variable potency. Proc. Natl. Acad. Sci. USA 2010, 107, 4335-4340. [CrossRef] [PubMed]

15. Hospodiuk, M.; Dey, M.; Sosnoski, D.; Ozbolat, I.T. The bioink: A comprehensive review on bioprintable materials. Biotechnol. Adv. 2017, 35, 217-239. [CrossRef] [PubMed]

16. Robinson, M.; Douglas, S.; Michelle Willerth, S. Mechanically stable fibrin scaffolds promote viability and induce neurite outgrowth in neural aggregates derived from human induced pluripotent stem cells. Sci. Rep. 2017, 7, 6250. [CrossRef] [PubMed]

17. Kim, I.-Y.; Seo, S.-J.; Moon, H.-S.; Yoo, M.-K.; Park, I.-Y.; Kim, B.-C.; Cho, C.-S. Chitosan and its derivatives for tissue engineering applications. Biotechnol. Adv. 2008, 26, 1-21. [CrossRef] [PubMed]

18. Tasnim, N.; De la Vega, L.; Kumar, S.A.; Abelseth, L.; Alonzo, M.; Amereh, M.; Joddar, B.; Willerth, S.M. 3D Bioprinting Stem Cell Derived Tissues. Cell. Mol. Bioeng. 2018, 11, 219-240. [CrossRef]

19. Mandrycky, C.; Wang, Z.; Kim, K.; Kim, D.-H. 3D bioprinting for engineering complex tissues. Biotechnol. Adv. 2016, 34, 422-434. [CrossRef] [PubMed]

20. Tasoglu, S.; Demirci, U. Bioprinting for stem cell research. Trends Biotechnol. 2013, 31, 10-19. [CrossRef] [PubMed]

21. Bsoul, A.; Cretu, E.; Walus, K. Lab-on-a-printer platform technology. In Proceedings of the 19th International Conference on Miniaturized Systems for Chemistry and Life Sciences, Gyeongju, Korea, 25-29 October 2015.

22. Lab-On-A-Printer. Available online: https://www.aspectbiosystems.com/technology (accessed on 20 September 2018).

23. Takazawa, T.; Croft, G.F.; Amoroso, M.W.; Studer, L.; Wichterle, H.; MacDermott, A.B. Maturation of Spinal Motor Neurons Derived from Human Embryonic Stem Cells. PLoS ONE 2012, 7, e40154. [CrossRef] [PubMed]

24. Neely, M.D.; Litt, M.J.; Tidball, A.M.; Li, G.G.; Aboud, A.A.; Hopkins, C.R.; Chamberlin, R.; Hong, C.C.; Ess, K.C.; Bowman, A.B. DMH1, a Highly Selective Small Molecule BMP Inhibitor Promotes Neurogenesis of hiPSCs: Comparison of PAX6 and SOX1 Expression during Neural Induction. ACS Chem. Neurosci. 2012, 3, 482-491. [CrossRef] [PubMed] 
25. Du, Z.-W.; Chen, H.; Liu, H.; Lu, J.; Qian, K.; Huang, C.-L.; Zhong, X.; Fan, F.; Zhang, S.-C. Generation and expansion of highly pure motor neuron progenitors from human pluripotent stem cells. Nat. Commun. 2015, 6, 6626. [CrossRef] [PubMed]

26. Hu, B.-Y.; Zhang, S.-C. Differentiation of spinal motor neurons from pluripotent human stem cells. Nat. Protoc. 2009, 4, 1295. [CrossRef] [PubMed]

27. De la Vega, L.; Karmirian, K.; Willerth, S.M. Engineering Neural Tissue from Human Pluripotent Stem Cells Using Novel Small Molecule Releasing Microspheres. Adv. Biosyst. 2018. [CrossRef]

28. Robinson, M.; Yau, S.-Y.; Sun, L.; Gabers, N.; Bibault, E.; Christie, B.R.; Willerth, S.M. Optimizing Differentiation Protocols for Producing Dopaminergic Neurons from Human Induced Pluripotent Stem Cells for Tissue Engineering Applications: Supplementary Issue: Stem Cell Biology. Biomarker Insights 2015, 10, BMI-S20064. [CrossRef]

29. Agbay, A.; De La Vega, L.; Nixon, G.; Willerth, S. Guggulsterone-releasing microspheres direct the differentiation of human induced pluripotent stem cells into neural phenotypes. Biomed. Mater. 2018, 13, 034104. [CrossRef] [PubMed]

30. Thomas, A.M.; Kubilius, M.B.; Holland, S.J.; Seidlits, S.K.; Boehler, R.M.; Anderson, A.J.; Cummings, B.J.; Shea, L.D. Channel density and porosity of degradable bridging scaffolds on axon growth after spinal injury. Biomaterials 2013, 34, 2213-2220. [CrossRef] [PubMed]

31. Ghasemi-Mobarakeh, L.; Prabhakaran, M.P.; Morshed, M.; Nasr-Esfahani, M.-H.; Ramakrishna, S. Electrospun poly ( $\varepsilon$-caprolactone)/gelatin nanofibrous scaffolds for nerve tissue engineering. Biomaterials 2008, 29, 4532-4539. [CrossRef] [PubMed]

32. Schwartz, M.P.; Hou, Z.; Propson, N.E.; Zhang, J.; Engstrom, C.J.; Costa, V.S.; Jiang, P.; Nguyen, B.K.; Bolin, J.M.; Daly, W. Human pluripotent stem cell-derived neural constructs for predicting neural toxicity. Proc. Natl Acad. Sci. USA 2015, 112, 12516-12521. [CrossRef] [PubMed]

33. Joung, D.; Truong, V.; Neitzke, C.C.; Guo, S.Z.; Walsh, P.J.; Monat, J.R.; Meng, F.; Park, S.H.; Dutton, J.R.; Parr, A.M. 3D Printed Stem-Cell Derived Neural Progenitors Generate Spinal Cord Scaffolds. Adv. Funct. Mater. 2018, 28, 1801850. [CrossRef]

34. Gu, Q.; Tomaskovic-Crook, E.; Lozano, R.; Chen, Y.; Kapsa, R.M.; Zhou, Q.; Wallace, G.G.; Crook, J.M. Functional 3D neural mini-tissues from printed gel-based bioink and human neural stem cells. Adv. Healthc. Mater. 2016, 5, 1429-1438. [CrossRef] [PubMed]

35. Gu, Q.; Tomaskovic-Crook, E.; Wallace, G.G.; Crook, J.M. 3D bioprinting human induced pluripotent stem cell constructs for in situ cell proliferation and successive multilineage differentiation. Adv. Healthc. Mater. 2017, 6, 1700175. [CrossRef] [PubMed] 